\title{
ANASTOMOSE COLÔNICA COM ADESIVO DE FIBRINA EM RATOS DIABÉTICOS
}

\author{
COLONIC ANASTOMOSIS WITH FIBRIN GLUE IN DIABETIC RATS
}

\author{
Orlando Ribeiro Prado Filho, TCBC-PR' ${ }^{\text {; }}$ Carlos Edmundo Rodrigues Fontes, TCBC-PR'; \\ Amélia Cristina Seidel'; Monice Dall'Ago Tomasi²; Heloise Dall'Ago Tomasi ${ }^{2}$
}

\begin{abstract}
RESUMO: Objetivo: Estudar aspectos da cicatrização na anastomose colônica com adesivo de fibrina em ratos normais e diabéticos. Método: Foram utilizados 160 ratos Wistar (Rattus novergicus albinus), machos, com peso variando de 250 a $300 \mathrm{~g}$, sendo que oitenta destes animais eram não diabéticos e os outros oitenta animais apresentavam diabetes mellitus induzido pela aloxana, Os animais foram submetidos a procedimento operatório padronizado que consistiu em colectomia parcial esquerda e anastomose primária por sutura manual ou com adesivo de fibrina. Foram reavaliados no quarto ou sétimo dia de pós-operatório quando se observou a presença de coleção líquida ou purulenta na tela subcutânea; integridade da sutura; presença de aderências; presença de deiscência; resistência da anastomose (pressão máxima de insuflação) em mmHg, e a concentração tecidual de hidroxiprolina. Resultados: Em relação à presença de deiscência de anastomose e mortalidade ocorreram piores resultados nos animais diabéticos no quarto e sétimo dias de observação em comparação ao controle. Nos grupos e subgrupos estudados os resultados com o uso do adesivo de fibrina não foram melhores que os da sutura manual. Em relação à concentração tecidual de hidroxiprolina não houve diferença significativa entre os grupos. Conclusão: O diabetes mellitus induzido pela aloxana impede a reparação normal da anastomose colônica em ratos e o uso adesivo de fibrina na anastomose colônica em ratos normais e diabéticos não trouxe benefícios na evolução pós-operatória (Rev. Col. Bras. Cir. 2008; 35(5): 329-333).
\end{abstract}

Descritores: Anastomose Cirúrgica; Adesivo tecidual de Fibrina; Colo; Diabetes mellitus; Ratos.

\section{INTRODUÇÃO}

As anastomoses no aparelho digestório obedecem a alguns princípios técnicos fundamentais. A técnica operatória de reconstrução do trânsito alimentar deve permitir que a anastomose seja continente, permeável, não propiciar isquemia na linha de sutura, afrontar as bordas da cicatriz sem causar estenose, produzir a menor intensidade de aderências possível e respeitar a comodidade visceral ${ }^{1}$.

Vários tipos de materiais e técnicas foram idealizados para tornar uma anastomose do trato digestório mais segura e eficaz. Fios cirúrgicos mais resistentes e inertes, com menor capacidade de desencadear reação inflamatória, tais como o ácido poliglicólico, a poliglactina 910 e o polipropileno ${ }^{2}$; grampeadores para sutura mecânica ${ }^{3}$; anéis biofragmentáveis ${ }^{4}$ e adesivos ${ }^{5,6}$ têm sido usados contemporaneamente.

Dentre os adesivos destaca-se o de fibrina, uma substância formada pelo fibrinogênio humano e aprotinina aos quais são acrescentados a trombina e o cloreto de cálcio. Ela contribui favoravelmente para a cicatrização da ferida por produzir hemostasia local e estimular o fluxo de macrófagos com a produção de fatores que favorecem a angiogênese, a proliferação de fibroblastos e a produção de colágeno $0^{5,7}$. Outros trabalhos experimentais, entretanto, mostraram resultados insatisfatórios, relatando o aumento da reação inflamatória e o retardo na cicatrização de anastomoses intestinais ${ }^{6,8-10}$.
Os mecanismos que levam o diabetes mellitus a impedir uma cicatrização adequada das feridas operatórias no intestino ainda não estão totalmente elucidadas ${ }^{11,12}$. Um grande número de evidências aponta que o estado diabético é associado com uma redução ou atraso da capacidade de reparação do tecido $^{12}$. Os pacientes diabéticos são mais susceptíveis à infecções que os pacientes normais, apresentando processos infecciosos não habituais como pielonefrite, colecistite aguda enfisematosa e fasciite necrotizante entre outros ${ }^{13}$.

O objetivo deste trabalho foi estudar aspectos da cicatrização anastomose colônica com adesivo de fibrina em ratos normais e diabéticos.

\section{MÉTODO}

Foram utilizados 160 ratos Wistar (Rattus novergicus albinus), machos, com peso variando de $250 \mathrm{~g}$ a $300 \mathrm{~g}$, com idade média de três meses, procedentes do Biotério Central da Universidade Estadual de Maringá (UEM), mantidos e operados no Laboratório de Inflamação do Departamento de Farmácia e Farmacologia da UEM onde permaneceram em observação no período pós-operatório. Os animais foram distribuídos aleatoriamente em dois grupos (Figura 1). Foram seguidos os princípios éticos para uso de animais de laboratório da Sociedade Brasileira de Ciência em Animais de Laboratório.

1. Professor Adjunto da Área de Clínica Cirúrgica do Departamento de Medicina da Universidade Estadual de Maringá - Maringá - PR.

2. Acadêmica de Medicina da Universidade Estadual de Maringá - Maringá - PR.

Recebido em 02/05/2008

Aceito para publicação 19/07/2008

Conflito de interesse: nenhum

Fonte de financiamento: nenhuma

Trabalho realizado na Universidade Estadual de Maringá - PR. 


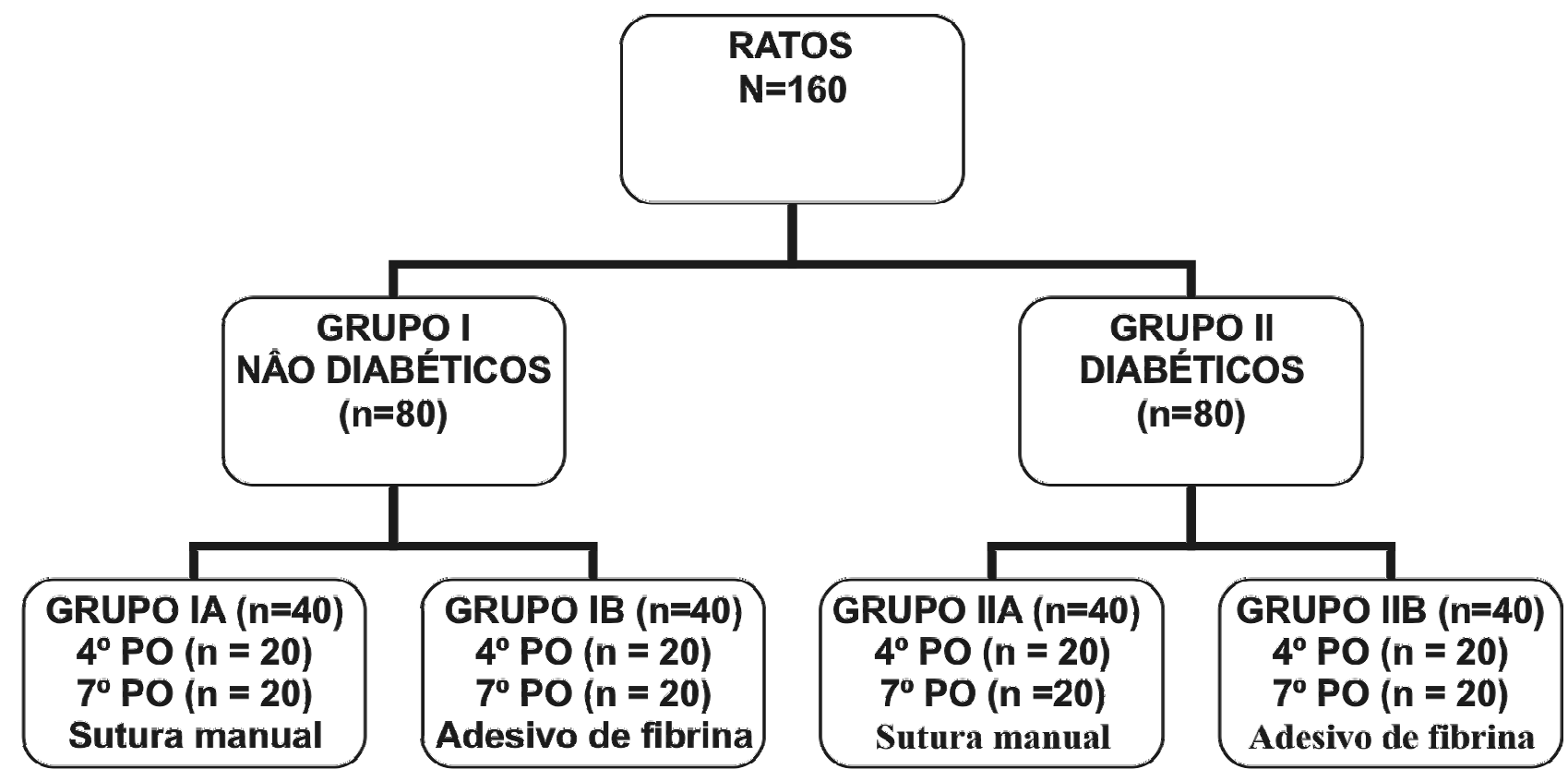

Figura 1 - Distribuição dos animais nos diversos grupos e subgrupos do estudo, segundo o tipo de aproximação dos cotos e tempo de observação no período pós-operatório.

O diabetes mellitus foi induzido pela administração de monoidrato de aloxana em solução salina na diluição de 45 mg.ml ${ }^{-1}$, em única dose de $45 \mathrm{mg} . \mathrm{kg}^{-1}$ de peso corporal, por via endovenosa, realizada na veia caudal. A confirmação da hiperglicemia foi realizada no $6^{\circ}$ dia após indução pela aloxana, colhendo sangue da veia caudal do animal, sempre no período vespertino. A determinação da glicemia foi realizada pelo método da glicose-oxidade-peroxidade. Foram incluídos no estudo somente os animais com glicemia igual ou superior a 200 mg. $\mathrm{dl}^{-1}$.

\section{Procedimento operatório}

Os animais foram anestesiados com injeção intramuscular da associação de xilazina e quetamina na relação de $1: 1$, na dose de $0,1 \mathrm{ml} \cdot \mathrm{kg}^{-1}$ de peso corporal Após anestesiados, os animais foram submetidos à depilação do local a ser operado e colocados numa goteira cirúrgica em decúbito dorsal. O procedimento operatório foi realizado com ventilação espontânea. Foi feita uma incisão longitudinal e mediana na parede ventral do abdome do animal, até a visibilização do intestino grosso. A uma distância proximal de $2,5 \mathrm{~cm}$ da reflexão peritoneal foi realizada a retirada de todos os planos de um segmento de $1,0 \mathrm{~cm}$ do cólon esquerdo (Figura 2).

Para aproximação dos cotos cranial e caudal foram aplicadas duas técnicas diferentes de anastomose: Grupo IA anastomose por sutura com pontos separados e um plano com fio de náilon 6-0. Grupo IB - anastomose com cola de fibrina, com aproximação prévia dos cotos cranial e caudal por quatro pontos eqüidistantes com fio de náilon 6-0. Grupo IIA - anastomose por sutura com pontos separados e um plano com fio de náilon 6-0, em ratos com diabetes mellitus induzido. Grupo IIB - anastomose com cola de fibrina, com aproxi- mação prévia dos cotos cranial e caudal por quatro pontos eqüidistantes com fio de náilon 6-0, em ratos com diabetes mellitus induzido.

Após a anastomose a fáscia e pele foram aproximadas em separado usando sutura contínua com fio de náilon 40 . Finalizado o ato operatório os animais foram mantidos em ambiente enriquecido com oxigênio por 30 minutos até a recuperação anestésica, e então encaminhados para os alojamentos.

\section{Pós-operatório}

No $4^{\circ}$ ou $7^{\circ}$ dia de pós-operatórios os animais foram anestesiados, com a técnica descrita anteriormente, e realizada nova laparotomia para retirada do segmento do cólon esquerdo que continha a anastomose (Figura 3). A morte ocorreu com o animal ainda anestesiado por exsanguinação pela secção da artéria aorta.

\section{DEISCENCIA ANASTOMOSE}

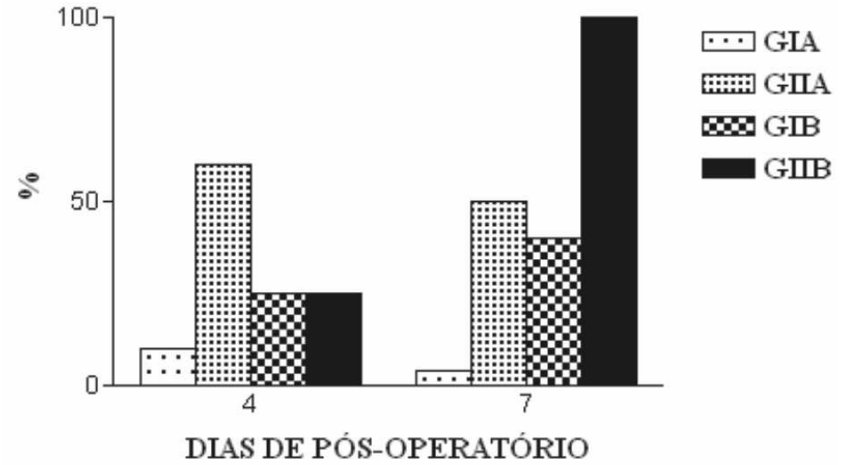

Figura 2 - Deiscência de anastomose nos diverso grupos no $4^{\circ}$ e $7^{o}$ dias de pós-operatório. 


\section{Avaliação macroscópica}

Para a avaliação da cicatriz operatória levou-se em conta a presença de coleção líquida ou purulenta na tela subcutânea e integridade da sutura. Em relação à cavidade abdominal observou-se a presença de aderências, para tanto se adotou o seguinte escore: Zero (0) - sem aderências; 1 (+) aderências mínimas, principalmente entre a anastomose e o grande omento ( desfeita com manobra digital) ; 2 (+) - aderências moderadas entre o omento e o local da anastomose ou entre a anastomose e a alça de intestino delgado (facilmente desfeita com tesoura); 3 (+) - intensa e extensa adesão (dificilmente desfeita com tesoura ).

Em relação à anastomose avaliou-se a presença de deiscência, ou seja, presença de solução de continuidade na linha de sutura, sem bloqueio, que permitisse a passagem de conteúdo entérico.

A concentração tecidual de hidroxiprolina foi mensurada no primeiro e segundo atos operatórios, no Laboratório de Inflamação do Departamento de Farmácia e Farmacologia da UEM, sendo a concentração expressa em mg.mg ${ }^{-1}$ de peso seco da amostra.

Para avaliação estatística foram utilizados o teste de Mann-Whitney e a análise de variância por postos de KruskalWallis. O nível de rejeição da hipótese de nulidade foi fixado em 0,05 ou $5 \%(\alpha \leq 0,05)$.

\section{RESULTADOS}

Em relação à presença de coleção purulenta na parede abdominal não houve diferença estatística da sua presença entre os grupos. Todos os animais apresentaram ao final do tempo de observação integridade da sutura da parede abdominal. Em todos os subgrupos houve ocorrência de aderências, sendo que as mais freqüentes foram tipo $1 \mathrm{e}$ 2. Não houve diferença estatística. Em relação à presença de deiscência de anastomose (Figura 4) e mortalidade (Figura 5) houve uma acentuada diferença para pior no grupo GIIA no quarto dia e GIIB no sétimo dia pós-operatório. Em relação à concentração tecidual de hidroxiprolina encontrada nas amostras colhidas na reoperação não houve diferença significativa nos animais sobreviventes na compara- ção entre os grupos GIA e GIB e nem entre os grupos GIIA e GIIB (Figura 6).

\section{DISCUSSÃO}

A presença do diabetes mellitus leva a um comprometimento da cicatrização das feridas operatórias. Acredita-se que a redução ou retardo no desenvolvimento da resistência da sutura seja uma causa potencial das complicações cirúrgi$\operatorname{cas}^{12}$.

Apesar dos avanços das técnicas operatórias e do material cirúrgico as deiscências nas anastomoses no intestino grosso ainda são alarmantes, ocorrendo entre $3 \%$ a $20 \%$ dos $\operatorname{casos}^{14}$. Isto continua sendo um grande desafio para os cirurgiões, pois, além da morbidade que causa, é responsável por um terço dos óbitos nas ressecções anteriores de reto ${ }^{15}$.

Apesar do adesivo de fibrina ter sido objeto de trabalhos experimentais e clínicos em vários tipos de anastomoses, ainda há dúvidas da indicação do seu uso em segmentos do tubo digestório com maior risco de deiscência ${ }^{16}$. Este é um produto desenvolvido com o intuito de promover a vedação de suturas, oclusão de fístulas, oclusão de cavidades de abscessos e prevenção de aderências no interior da cavidade peritoneal5,6. Vários autores utilizaram-na como complemento em suturas do intestino delgado ${ }^{5,10,17}$ e grosso $^{8,9,17}$, com resultados conflitantes quanto à sua eficiência na proteção das anastomoses. Outros autores utilizaram-no como proteção em situações adversas para a feitura da anastomose ${ }^{18-20}$.

$\mathrm{O}$ adesivo de fibrina é composto por dois componentes, que misturados, resultam em um vedante. O fibrinogênio é a maior porção do primeiro componente, que também contém o fator XIII e proteínas plasmáticas. O segundo componente consiste em trombina, cloreto de cálcio e aprotinina (agente antifibrinolítico). A aprotinina previne a lise prematura do coágulo de fibrina e a trombina converte fibrinogênio em fibrina, dando início ao processo de solidificação da solução. Na presença do cloreto de cálcio, a trombina ativa a fator XIII que catalisa a formação das ligações da fibrina, o que intensifica a força tênsil do coágulo ${ }^{5,6}$.

Vários autores ${ }^{21-23}$ têm referido que o adesivo de fibrina pela sua composição poderia ser um fator favorável para o

\section{MORTALIDADE}

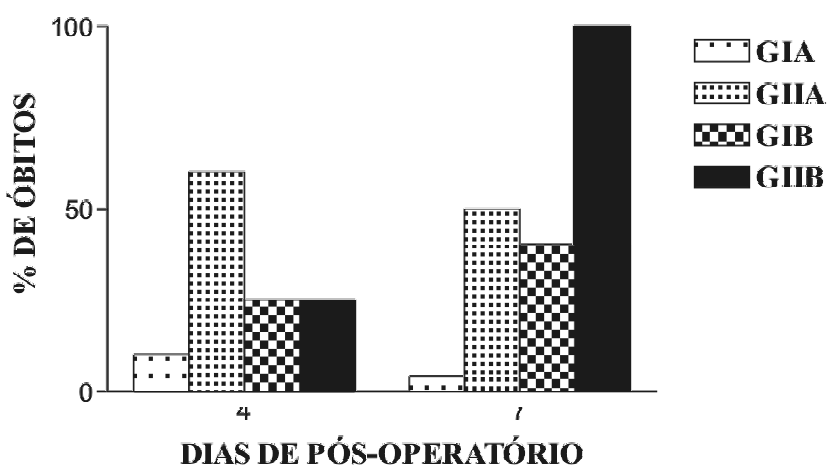

Figura 3 - Mortalidade entre os diversos grupos no $4^{\circ}$ e $7^{\circ}$ dias de pós-operatório.

\section{HIDROXIPROLINA}

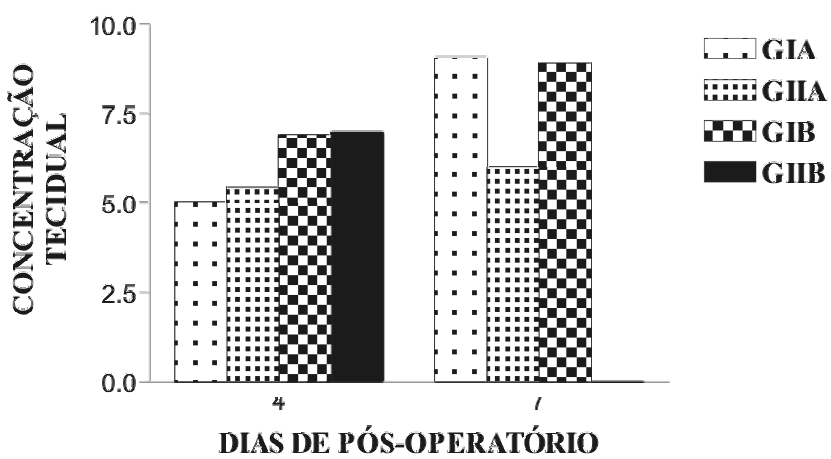

Figura 4-Concentração tecidual de hidroxiprolina em fragmento da linha de anastomose nos diversos grupos no $4^{\circ}$ e $7^{\circ}$ dias de pós-operatório. 
desenvolvimento de infecção, principalmente em situações de procedimentos contaminados. Neste trabalho também se observou desvantagens com o seu uso, aparentemente este produto foi um fator que piorou a evolução dos animais no período pós-operatório, pois a sua presença foi a causa provável das complicações infecciosas, das falhas de cicatrização das suturas e óbitos (Figuras 4 e 5). Capitán Morales et $a l{ }^{7}$ fizeram referência sobre a menor resistência à pressão de ruptura em anastomose feitas com adesivo de fibrina, em comparação ao uso de fio inabsorvível.

A hidroxiprolina é um aminoácido que corresponde a $14 \%$ da quantidade do colágeno total e há uma relação direta entre ela e a cicatrização da anastomose ${ }^{23}$. A concentração tecidual de colágeno na região da anastomose e suas variações no período pós-operatório dependem do material e técnica operatória usada ${ }^{24-28}$. Verhofstad e Hendriks ${ }^{11}$ demonstraram que o diabetes mellitus impede o desenvolvimento normal da anastomose intestinal, porém isto não foi causado pela redução da concentração tecidual de colágeno no local da sutura. Neste experimento as diferenças não foram significantes no quarto dia de observação. Já no sétimo dia de observação a concentração foi maior nos grupos apenas com sutura manual. Isto no leva a crer que no segundo período de observação a presença do adesivo de fibrina foi um fator deletério para a reparação da sutura.

Concluindo, o diabetes mellitus induzido pela aloxana impede a reparação normal da anastomose colônica em ratos. $\mathrm{O}$ adesivo de fibrina é um fator de piora na reparação da anastomose colônica em ratos normais e diabéticos.

\begin{abstract}
Background: The aim of this experimental study was to assess healing in colonic anastomosis with fibrin glue in normal and diabetics rats. Methods: One hundred and sixty Wistar rats were randomly assigned for two groups (normal and diabetics). The animals underwent a partial left colectomy and after an end-to-end anastomosis or a fibrin glue anastomosis. The rats were submitted to a new operative procedure on the 4th or 7th postoperative day, when the anastomosis were observed. The bursting pressure measurements were recorded. Results: Mortality and anastomosis dehiscence were greater in diabetic animals on 4th and 7th days of observation when compared to controlled rats. There have been no differences between groups and sub-groups regarding the use of fibrine patch or handmade suture. There were no significant differences on hidroxiproline tissue concentration. Conclusions: Aloxane-induced Diabetes mellitus impairs normal healing in colonic anastomosis in rats, and there were no benefits in normal or diabetic groups with the use of fibrin glue.
\end{abstract}

Key words: Anastomosis, surgical; Fibrin tissue adhesive; Colon; Diabetes mellitus; Rats.

\section{REFERÊNCIAS}

1. Fagundes DJ, Goldenberg S. Cirurgia geral. Pré e pós-operatório. São Paulo: Atheneu; 1995.

2. Fagundes DJ, Kharmandayan P. O fio cirúrgico. Acta Cir Bras. 1991; 6(3):177-81.

3. Steinchen FM, Ravitch MM. Instrumentos de grampeamento contemporâneo e

técnicas básicas de sutura mecânica. Clin Cir Am Norte. 1994; 3:445-62.

4. Hardy TG Jr, Pace WG, Maney JW, Katz AR, Kaganov AL. A biofragmentable ring for sutureless bowel anastomosis. An experimental study. Dis Colon Rectum. 1985; 28(7):484-90.

5. Sousa C. Uso de adesivos biológicos em anastomoses intestinais - estudo experimental em coelhos [dissertação]. Belo Horizonte (MG): Universidade Federal de Minas Gerais; 1993.

6. Prado Filho OR, Fagundes DJ, Nigro AJT, Bandeira COP, Novo NF, Juliano Y. Estudo morfológico da anastomose esôfagoesofágica cervical com adesivo de fibrina, em cães. Acta Scientiarum. 2002; 24(3):697-701.

7. Capitán Morales LC, Nuñez ER, Morales Conde S, Sanchez Ganfornina F, Del Rio Lafuente FD, Cabot Ostos E, Ortega Beviá JM, Loscertales Abril J, Cantillana Martínez J. Experimental study of sutureless colorectal anastomosis. Hepatologastrenterology. 2000; 47(35):1284-90.

8. van der Ham AC, Kort WJ, Weijma IM, Jeekel H. Transient protection of incomplete colonic anastomoses with fibrin sealant: an experimental study in the rat. J Surg Res. 1993; 55(3):256-60.
9. van der Ham AC, Kort WJ, Weijma IM, van den Ingh HF, Jeekel H. Effect of fibrin sealant on the integrity of colonic anastomoses in rats with faecal peritonitis. Eur J Surg. 1993; 159(8):425-32.

10. Trignano M, Pisano I, Mastino GP, Sini G, Bresadola V, de Anna D, Tanda F, Cossu-Rocca P, Canu L, Tolu E. Suture senza suture in chirurgia digestiva. Studio sperimentale sull'intestino del ratto. Ann Ital Chir. 1996; 67(3):419-23; discussion 423-4.

11. Verhofstad MH, Hendriks T. Diabetes impairs the development of early strength, but not the accumulation of collagen, during intestinal anastomotic healing in the rat. Br J Surg. 1994; 81(7):1040-5.

12. Verhofstad MH, Lange WP, van der Laak JA, Verhofstad AA, Hendriks T. Microscopic analysis of anastomotic healing in the intestine of normal rats. Dis Colon Rectum. 2001;44(3):423-31.

13. Seidel AC, Fagundes DJ, Bazotte RB, Novo NF, Juliano Y, Meister $\mathrm{H}$. Effect of lung resection and sham surgery on the frequency of infection in alloxan-diabetic rats. Braz J Med Biol Res. 2003; 36(3):287-90.

14. Tocchi A, Mazzoni G, Lepre L, Costa G, Liotta G, Agostini N, Miccini M. Prospective evaluation of omentoplasty in preventing leakage of colorectal anastomosis. Dis Colon Rectum. 2000; 43(7):951-5.

15. Santos Júnior JC. Profilaxia das complicações pós-operatórias no tratamento cirúrgico das doenças do intestino grosso: II Deiscência da anastomose. Rev Bras Colo-proctol. 1998; 18(1):44-51.

16. Karahasanoglu T, Alcicek S, Altunkaya E, Sahinler I, Goksel S, Sirin F, Ozbal A. Effect of fibrin glue on irradiated colonic anastomoses. Dis Colon Rectum. 1997; 40(10):1240-3. 
17. Shinohara K, Kobayashi E, Yoshida T, Toyama N, Kiyozaki H, Fujimura A, Miyata M. Effect of fibrin glue on small and large bowel anastomoses in the rat. Eur Surg Res. 1997; 30(1):8-12.

18. Kanellos I, Mantzoros I, Demetriades H, Kalfadis S, Kelpis T, Sakkas L, Betsis D. Healing of colon anastomoses covered with fibrin glue after immediate postoperative intraperitoneal administration of 5-fluorouracil. Dis Colon Rectum. 2004; 47(4):510-5.

19. Fontes CER, Taha MO, Fagundes DJ, Prado Filho O, Ferreira MV, Mardegan MJ. Estudo do reparo do ferimento de colon com o lado seroso da parede de jejuno utilizando cianoacrilato e cola de fibrina. Rev Col Bras Cir. 2006; 33(1):68-73.

20. Kanellos D, Blouhos K, Pramateftakis MG, Kanellos I, Demetriades H, Sakkas L, Betsis D. Effect of 5-fluorouracil plus interferon on the integrity of colonic anastomoses covering with fibrin glue. World J Surgery. 2007; 31(1):186-91.

21. Ertekin C, Kurdoglu M, Ozgur M. The healing of intestinal anastomosis and the effect of suture materials. Turk J Surg. 1989; $5(1): 31-4$.

22. Spotnitz WD, Falstrom JK, Rodeheaver GT. The role of sutures and fibrin sealant in wound healing. Surg Clin North Am. 1997; 77(3):651-69.

23. Uzunköy A, Akinci OF, Coskun A, Aslan O, Kocyigit A. Effects of antiadhesive agents on the healing of intestinal anastomosis. Dis Colon Rectum. 2000; 43(3):370-5.

24. Jiborn $\mathrm{H}$, Ahonen J, Zederfeldt B. Healing of experimental colonic anastomoses. I. Bursting strength of the colon after left colon resection and anastomosis. Am J Surg. 1978; 136(5):587-94.
25. Jiborn H, Ahonen J, Zederfeldt B. Healing of experimental colonic anastomoses. II. Bursting strength of the colon after left colon resection and anastomosis. Am J Surg. 1978; 136(5):595-9.

26. Jiborn H, Ahonen J, Zederfeldt B. Healing of experimental colonic anastomoses. III. Collagen metabolism in the colon after left colon resection. Am J Surg. 1980; 139(3):398-405.

27. Jiborn H, Ahonen J, Zederfeldt B. Healing of experimental colonic anastomoses. IV. Effect of suture technique on collagen metabolism in the colonic wall. Am J Surg. 1980; 139(3):40613 .

28. Prado Filho OR, Fagundes DJ, Nigro AJT, Bandeira COP, Novo $\mathrm{N}$, Juliano Y. Uso do adesivo de fibrina na anastomose esôfagoesofágica cervical, em cães. Rev Col Bras Cir. 2004; 31(4):22832 .

Como citar este artigo:

Prado Filho OR, Fontes CE, Seidel AC, Tomasi MD, Tomasi HD. Anastomose colônica com adesivo de fibrina em ratos diabéticos. Rev Col Bras Cir. [periódico na Internet] 2008; 35(5). Disponível em URL: http://www.scielo.br/rcbc

Endereço para correspondência:

Orlando Ribeiro Prado Filho

Rua Arthur Thomaz, 23 - ap. 1102

87013-250 - Maringá - PR

E-mail: orlandorpf@hotmail.com 